\title{
Convergence of the ACM Finite Element Scheme for Plate Bending Problems
}

\author{
By \\ Fumio KIKUCHI*
}

\begin{abstract}
Summary
Convergence rates of the ACM non-conforming scheme are evaluated. This scheme is usually employed for two-dimensional bi-harmonic boundary-value and eigenvalue problems arising from plate bending analysis. When the shape of the domain is rectangular and the exact solution is sufficiently smooth, $L_{2}$-error bounds of moments and deflection and error of eigenvalue are all at the order of square of the maximum mesh size. This result is also confirmed by numerical experiments.
\end{abstract}

\section{Introduction}

In the engineering literatures, non-conforming finite elements are frequently employed especially for plate bending problems (Zienkiewicz, [11]). In this type of approximation, the trial functions do not belong to the energy space, and the finite element solution may or may not converge to the exact solution. The mathematical studies of this method have been conducted, for example, by Strang and Fix [10], Babuska and Zlamal [1], and Ciarlet [2]. However, the case study appears to be still insufficient since the convergence depends strongly upon the specific features of the individual schemes. This paper deals with an important non-conforming scheme in practice.

The ACM scheme is one of the most popular finite element scheme for plate bending (see Melosh [5]). Although it is non-conforming, the accuracy of the solutions obtained by its use is known to be excellent. In fact, we can readily find the following statement in Zienkiewicz [11]: The linear distribution of moments tries, as it were,

Communicated by S. Matsuura, January 20, 1975.

* Institute of Space and Acronautical Science, University of Tokyo, Tokyo. 
to give the "best fit" to the exact moment distribution at all stage of the subdivisions.

As for its convergence for boundary-value problems, Miyoshi [7] performed an error analysis for clamped plates subdivided into regular meshes. His main result is that the orders of errors of the deflection and the moments (second-order derivatives of deflection) are square root of the maximum mesh size $h$. When the shape of the domain is exactly represented by this rectangular element and the exact solution is sufficiently smooth, the orders may be improved up to $O(h)$, as is seen from his analysis.

On the other hand, we can presume that the orders would be $O\left(h^{2}\right)$ in certain cases, if the Zienkiewicz observation is true. The aim of this paper is to derive some error bounds to the finite element approximation of both boundary-value and eigenvalue problems of simply supported rectangular plates, from which we can see that the above conjecture is true. To this end, the scheme is regarded as an improved one of Melosh's scheme [4] based on the partial approximation. Numerical experiments are also conducted for a few simple problems to see the validity of the theory.

\section{Preliminaries}

Let $\boldsymbol{R}^{2}$ be the two-dimensional Euclidean space, a point of which is designated by $x=\left(x_{1}, x_{2}\right)$, and $\Omega \subset \boldsymbol{R}^{2}$ is a rectangular domain defined by $\left|x_{i}\right|<d_{i} / 2(i=1,2)$. In the sequel, $C, C^{*}, C_{1}$ etc. are generic positive constants independent of various parameters and may take different values when appear in different places.

Let $H^{n}(\Omega)$ be the usual $n$-th order real Sobolev space with $n$ being a non-negative integer. The norm of $u \in H^{n}(\Omega)$ is given by

$$
\|u\|_{n, \Omega}=\left(\sum_{|\alpha| \leqq n} \int_{\Omega}\left|D^{\alpha} u(x)\right|^{2} d x\right)^{\frac{1}{2}}
$$

where $\alpha=\left(\alpha_{1}, \alpha_{2}\right)$ is a two-component index, $\alpha_{i}$ 's being non-negative integers, $|\alpha|=\alpha_{1}+\alpha_{2}$ and $D^{\alpha}=\partial^{|\alpha|} / \partial x_{1}^{\alpha} \partial x_{2}^{\alpha}$. The space $H_{0}^{n}(\Omega)$ is the completion, with respect to the norm (1), of the space of all test func- 
tions on $\Omega$. For $n=0, H^{0}(\Omega)=H_{0}^{0}(\Omega)$ is the usual $L_{2}(\Omega)$ space, the inner product and the norm of which are respectively denoted by $(,)_{\Omega}$ and \|\|$_{\Omega}$. We will also use a semi-norm of $H^{n}(\Omega)$ defined by

$$
|u|_{n \Omega}=\left(\sum_{|\alpha|=n} \int_{\Omega}\left|D^{\alpha} u(x)\right|^{2} d x\right)^{\frac{1}{2}} \text {. }
$$

Let us consider a biharmonic operator $A=\Delta \Delta$ with the domain of definition

$$
D(A)=\left\{u \mid u \in C^{4}(\bar{\Omega}) \quad \text { and } \quad u=\partial^{2} u / \hat{o} n^{2}=0 \quad \text { on } \quad \partial \Omega\right\}
$$

where $\bar{\Omega}$ is the closure of $\Omega, \partial \Omega$ the boundary of $\Omega$, and $\partial^{2} u / \partial n^{2}$ the second order derivative of $u$ in the outward normal direction of $\partial \Omega$. The boundary conditions in the above correspond to the simply supported edges of plates. Clearly, $D(A) \subset H^{4}(\Omega) \cap H_{0}^{1}(\Omega)$, and the range of $A$ is included into $L_{2}(\Omega)$. The use of the divergence theorem yields

$$
(A u, \bar{u})_{\Omega}=(u, A \bar{u})_{\Omega}=\sum_{i, j=1}^{2}\left(\partial^{2} u / \partial x_{i} \partial x_{j}, \partial^{2} \bar{u} / \partial x_{i} \partial x_{j}\right)_{\Omega}
$$

for any $u, \bar{u} \in D(A)$. Thus it is easy to show that $A$ is symmetric and satisfies (cf. Lemma 1 in this paper)

$$
\|u\|_{2, \Omega_{2}}^{2} \geqq(A u, u)_{\Omega} \geqq C\|u\|_{2}^{2}, \Omega \quad\left({ }^{\forall} u \in D(A)\right),
$$

where $C$ is a positive constant dependent only on $d_{1}$ and $d_{2}$.

Now we can use the standard procedure to obtain the energy space $H_{A}$ associated with $A$ (see sec. 9 of Mikhlin [6]): we first define the inner product $\langle$,$\rangle and the energy norm \|\| \|$ for the elements of $D(A)$ by

$$
\langle u, \bar{u}\rangle=(A u, \bar{u})_{\Omega} \quad \text { and } \quad\|u\|=\langle u, u\rangle^{\frac{1}{2}}
$$

and then obtain $H_{A}$ as the completion of $D(A)$ with respect to the energy norm. We will use the same notations as the above even for the inner product and the norm of $H_{A}$.

It is not difficult to show

$$
H_{A}=H^{2}(\Omega) \cap H_{0}^{1}(\Omega) .
$$


This may be done by first extending $u \in H^{2}(\Omega) \cap H_{0}^{1}(\Omega)$ outside $\Omega$ in an anti-symmetric manner with respect to $\partial \Omega$, and then using a sufficiently smooth symmetric mollifier to get an element of $D(A)$ arbitrarily close to $u$ in the norm of $H^{2}(\Omega)$. Consequently, we obtain the following explicit expressions of the inner product and the norm of $H_{A}$ :

$$
\begin{gathered}
\langle u, \bar{u}\rangle=\sum_{i, j=1}^{2}\left(\partial^{2} u / \partial x_{i} \partial x_{j}, \partial^{2} \bar{u} / \partial x_{i} \partial x_{j}\right)_{\Omega}, \\
\left.\|u\||=| u\right|_{2, \Omega}
\end{gathered}
$$

for any $u, \bar{u} \in H_{A}$.

A variational formulation for the static (boundary-value) problem of the plate is to find a deflection $u \in H_{A}$ for an arbitrarily given load $f \in L_{2}(\Omega)$ such that

$$
\langle u, \bar{u}\rangle=(f, \bar{u})_{\Omega} \quad\left({ }^{\forall} \bar{u} \in H_{A}\right),
$$

while that for the vibration (eigenvalue) problem is to find non-zero $u \in H_{A}$ and a real number $\lambda$ such that

$$
\langle u, \bar{u}\rangle=\lambda(u, \bar{u})_{\Omega} \quad\left({ }^{\forall} \bar{u} \in H_{A}\right) .
$$

Here we have assumed both the bending rigidity and the mass per unit area of the plate to be unity and Poisson's ratio to be zero.

The following two theorems are on the solutions of the above two problems.

Theorem 1. The solution of Eq. (5) exists uniquely in $H_{A}$ for any $f \in L_{2}(\Omega)$ and satisfies

$$
\begin{aligned}
& u \in H^{4}(\Omega), \quad\|u\|_{4, \Omega} \leqq C\|f\|_{\Omega}, \\
& u=\partial^{2} u / \partial n^{2}=0 \quad \text { on } \quad \partial \Omega .
\end{aligned}
$$

Therefore, $u$ satisfies $\Delta \Delta u=f$ in $\Omega$ in the strong sense, and the traces in Eq. (8) may be regarded as continuous functions on $\partial \Omega$.

Proof. The uniqueness and the existence follow from the Riesz representation theorem. The smoothness of $u$ may be established by 
constructing the solution explicitly by the Fourier double series. (cf. Theorem 1.10 of Mizohata [8] and Lemma 1 of Hall and Kennedy [3].)

The following is easy to check by the use of compactness theorems and the preceding theorem.

Theorem 2. The set of all eigenvalues for Eq. (6) is a countable set in $] 0, \infty[$ without any accumulation points (except at infinity). We can arrange the eigenvalues $\left\{\lambda_{i}\right\}_{i=1}^{\infty}$ in such a way that

$$
0<\lambda_{1} \leqq \lambda_{2} \leqq \lambda_{3} \leqq \cdots<\infty
$$

The corresponding eigenfunctions $\left\{u_{i}\right\}_{i=1}^{\infty}$ can be normalized as

$$
\left(u_{i}, u_{j}\right)_{\Omega}=\delta_{i j} \quad\left(\delta_{i j}: \text { Kronecker's delta }\right)
$$

and satisfy

$$
\begin{aligned}
& u_{i} \in H^{4}(\Omega), \quad\left\|u_{i}\right\|_{4, \Omega} \leqq C \lambda_{i} \\
& u_{i}=\partial^{2} u_{i} / \partial n^{2}=0 \quad \text { on } \quad \partial \Omega
\end{aligned}
$$

\section{Finite Element Schemes}

Let us decompose $\Omega$ into rectangular elements $\left\{\Omega_{h i}\right\}_{i=1}^{N_{h}}$ by lines parallel to the coordinate axes (see Fig. 1). The side lengths of $\Omega_{h i}$

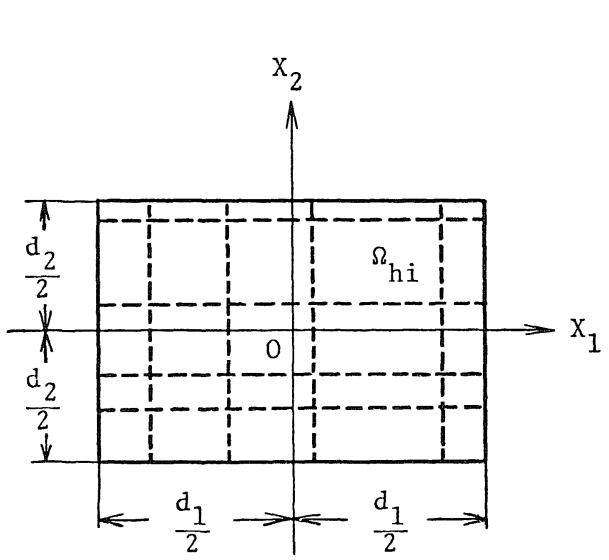

FINITE ELEMENT DECOMPOSITION

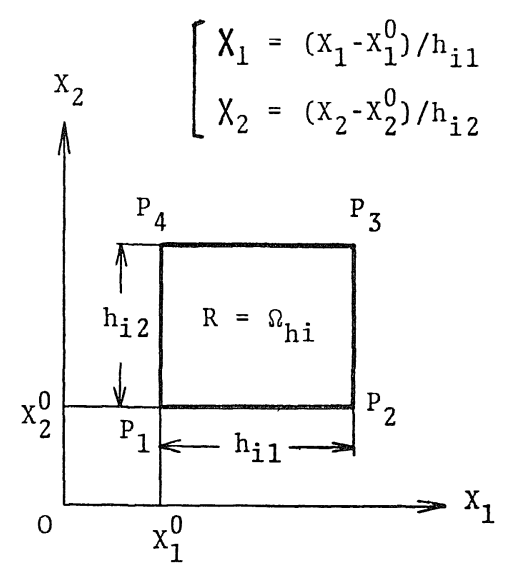

RECTANGULAR ELEMENT

Figure 1. Finite element decomposition of the domain into rectangular moshes. 
in $x_{1}$ and $x_{2}$ directions are respectively denoted by $h_{i 1}$ and $h_{i 2}$, for which we assume

$$
C_{1} \geqq h_{i 2} / h_{i 1} \geqq C_{2}>0,
$$

where $C_{1}$ and $C_{2}$ are pre-assigned positive constants. We also use the notations $h_{i}=\max \left\{h_{i 1}, h_{i 2}\right\}$ and $h=\max _{1 \leqq i \leqq N_{h}} h_{i}$.

In the ACM scheme, the distribution of the approximate function $u_{h}$ is given by linear combination of $x_{1}^{\alpha_{1}} x_{2}^{\alpha_{2}}$ with $0 \leqq \alpha_{i} \leqq 3$ and $|\alpha| \leqq 4$ (excluding the case $\alpha_{1}=\alpha_{2}=2$ ) in each element. Globally, it can be expressed by

$$
u_{h}(x)=\sum_{|\alpha| \leqq 1} \sum_{j=1}^{M_{h}} D^{\alpha} u_{h}\left(P_{j}\right) \phi_{h j}^{\alpha}(x)
$$

where $P_{j}$ is the $j$-th nodes of the mesh. Here, $u_{h}$ and its first derivatives are forced to be continuous at all nodes, and the shape function $\phi_{h j}^{\alpha}$ satisfies

$$
D^{\beta} \phi_{h j}^{\alpha}\left(P_{k}\right)= \begin{cases}1 & (\alpha=\beta \text { and } j=k), \\ 0 & \text { (otherwise) }\end{cases}
$$

for $|\alpha|,|\beta| \leqq 1$ and $1 \leqq j, k \leqq M_{h}$. When $P_{j} \in \partial \Omega$, some of the above nodal values vanish identically to satisfy the condition $u_{h}=0$ on $\partial \Omega$. The precise definition of $u_{h}$ may be found in page 177 of Zienkiewicz [11]. The finite element space thus defined is denoted by $S^{h}$. Although $S^{h} \subset H_{0}^{1}(\Omega)$ and $\partial^{2} u_{h} / \partial x_{1} \partial x_{2} \in L_{2}(\Omega)$ for any $u_{h} \in S^{h}, S^{h}$ is non-conforming, i.e. $S^{h} \notin H_{A}$ because $S^{h} \notin H^{2}(\Omega)$. However, the restriction of $u_{h} \in S^{h}$ to a finite element $\Omega_{h k}$ belongs to $H^{2}\left(\Omega_{h k}\right)$, so that the following bilinear form is well-defined for any $u_{h}, \bar{u}_{h} \in S^{h}$ and any $u, \bar{u} \in H^{2}(\Omega)$ (cf. Babuska and Zlamal [1]):

$$
\left\langle u_{h}+u, \bar{u}_{h}+\bar{u}\right\rangle_{h}=\sum_{k=1}^{N_{h}} \sum_{i, j=1}^{2}\left(\partial^{2}\left(u_{h}+u\right) / \partial x_{i} \partial x_{j}, \partial^{2}\left(\bar{u}_{h}+\bar{u}\right) / \partial x_{i} \partial x_{j}\right)_{\Omega_{h k}} .
$$

The following is also well-defined:

$$
\left\|u_{h}+u\right\|_{h}=\left\langle u_{h}+u, u_{h}+u\right\rangle_{h}^{\frac{1}{2}} .
$$


Particularly, $\langle u, \bar{u}\rangle_{h}=\langle u, \bar{u}\rangle$ and $\|u\|_{h}=\|u\| \|$.

Let us introduce two mappings $J_{h i}$ 's $(i=1,2)$ from $S^{h}$ into $H_{0}^{1}(\Omega)$. If we consider a typical rectangular element $R=\Omega_{h j}$ with nodes $P_{k}$ 's $(k=1,2,3,4)$, the distribution of $J_{h i} u_{h}$ 's for $u_{h} \in S^{h}$ are given in $R$ by

$$
\begin{aligned}
J_{h 1} u_{h}= & \left(1+2 X_{1}\right)\left(1-X_{1}\right)^{2}\left[\left(1-X_{2}\right) u_{h}\left(P_{1}\right)+X_{2} u_{h}\left(P_{4}\right)\right] \\
& +X_{1}^{2}\left(3-2 X_{1}\right)\left[\left(1-X_{2}\right) u_{h}\left(P_{2}\right)+X_{2} u_{h}\left(P_{3}\right)\right] \\
& +h_{j 1} X_{1}\left(1-X_{1}\right)^{2}\left[\left(1-X_{2}\right) D^{(1,0)} u_{h}\left(P_{1}\right)+X_{2} D^{(1,0)} u_{h}\left(P_{4}\right)\right] \\
& +h_{j 1} X_{1}^{2}\left(X_{1}-1\right)\left[\left(1-X_{2}\right) D^{(1,0)} u_{h}\left(P_{2}\right)+X_{2} D^{(1,0)} u_{h}\left(P_{3}\right)\right]
\end{aligned}
$$

$$
\begin{aligned}
J_{h 2} u_{h}= & \left(1+2 X_{2}\right)\left(1-X_{2}\right)^{2}\left[\left(1-X_{1}\right) u_{h}\left(P_{1}\right)+X_{1} u_{h}\left(P_{2}\right)\right] \\
& +X_{2}^{2}\left(3-2 X_{2}\right)\left[\left(1-X_{1}\right) u_{h}\left(P_{4}\right)+X_{1} u_{h}\left(P_{3}\right)\right] \\
& +h_{j 2} X_{2}\left(1-X_{2}\right)^{2}\left[\left(1-X_{1}\right) D^{(0,1)} u_{h}\left(P_{1}\right)+X_{1} D^{(0,1)} u_{h}\left(P_{2}\right)\right] \\
& +h_{j 2} X_{2}^{2}\left(X_{2}-1\right)\left[\left(1-X_{1}\right) D^{(0,1)} u_{h}\left(P_{4}\right)+X_{1} D^{(0,1)} u_{h}\left(P_{3}\right)\right]
\end{aligned}
$$

where $X_{1}$ and $X_{2}$ are local coordinates of $R$ defined in Fig. 1. Furthermore, it holds in $R$ that

$$
\begin{aligned}
u_{h} & =J_{h 1} u_{h}+J_{h 2} u_{h}-\left(1-X_{1}\right)\left(1-X_{2}\right) u_{h}\left(P_{1}\right)-X_{1}\left(1-X_{2}\right) u_{h}\left(P_{2}\right) \\
& -X_{1} X_{2} u_{h}\left(P_{3}\right)-\left(1-X_{1}\right) X_{2} u_{h}\left(P_{4}\right)
\end{aligned}
$$

We can see that $\partial^{2} J_{h i} u_{h} / \partial x_{i}^{2} \in L_{2}(\Omega)$ for any $u_{h} \in S^{h}$, and in $R$ holds the relation $\partial^{2} J_{h i} u_{h} / \partial x_{i}^{2}=\partial^{2} u_{h} / \partial x_{i}^{2}(i=1,2)$. Therefore, Eq. (15) may be rewritten by

$$
\begin{aligned}
\left\langle u_{h}+u, \bar{u}_{h}+\bar{u}\right\rangle_{h}= & \sum_{i=1}^{2}\left(\partial^{2}\left(J_{h i} u_{h}+u\right) / \partial x_{i}^{2}, \partial^{2}\left(J_{h i} \bar{u}_{h}+\bar{u}\right) / \partial x_{i}^{2}\right)_{\Omega} \\
& +2\left(\partial^{2}\left(u_{h}+u\right) / \partial x_{1} \partial x_{2}, \partial^{2}\left(\bar{u}_{h}+\bar{u}\right) / \partial x_{1} \partial x_{2}\right)_{\Omega} .
\end{aligned}
$$

We will present some lemmas to clarify the properties of $S^{h}$.

Lemma 1. For any $u_{h} \in S^{h}$ and $u \in H_{A}$ hold the inequalities

$$
\left\|u_{h}+u\right\|_{h} \geqq C\left\|D^{\alpha}\left(u_{h}+u\right)\right\|_{\Omega} \geqq C^{*}\left\|u_{h}+u\right\|_{\Omega},
$$




$$
\left\|\partial^{2}\left(u_{h}+u\right) / \partial x_{1} \partial x_{2}\right\|_{\Omega} \geqq C \max _{x \in \bar{\Omega}}\left|u_{h}(x)+u(x)\right|
$$

(19 c) $\quad\left\|\partial^{2}\left(J_{h i} u_{h}+u\right) / \partial x_{i}^{2}\right\|_{\Omega} \geqq C\left\|\partial\left(J_{h i} u_{h}+u\right) / \partial x_{i}\right\|_{\Omega} \geqq C^{*}\left\|J_{h i} u_{h}+u\right\|_{\Omega}$

for $|\alpha|=1$ and $i=1,2$.

Proof. See sec. 30 of Mikhlin [6].

Lemma 2. (uniformity condition) Let $\phi_{h j}^{\alpha}$ be the shape function in Eq. (14). Then it holds that

$$
\sup _{x \in \Omega_{h i}}\left|D^{\beta} \phi_{h j}^{\alpha}(x)\right| \leqq C h_{i}^{|\alpha|-|\beta|}
$$

for $|\beta| \leqq 2$, where $C$ can be taken independent of $i\left(1 \leqq i \leqq N_{h}\right)$ and $j$.

Proof. This follows from the condition (13) and the relation $0 \leqq X_{1}$, $X_{2} \leqq 1$.

Lemma 3. Let $\hat{u}_{h} \in S^{h}$ be the interpolate of $u \in H^{4}(\Omega) \cap H_{0}^{1}(\Omega)$. This is well-defined since $u$ is sufficiently smooth and any element of $S^{h}$ vanishes on $\partial \Omega$. Then

$$
\left\|\hat{u}_{h}-u\right\|_{h} \leqq C h^{2}|u|_{4, \Omega} .
$$

Proof. We can use the Bramble-Hilbert theorem to derive this estimate, together with the uniformity condition just established (see page 143 of Strang and Fix [10]).

Lemma 4. Any $u_{h} \in S^{h}$ satisfies the relation

$$
\left\|J_{h i} u_{h}-u_{h}\right\|_{\Omega} \leqq C h^{2}\left\|u_{h}\right\|_{h} \quad(i=1,2) .
$$

Proof. As seen from Eq. (17), $J_{h i} u_{h}$ is precisely the piecewise linear interpolation of $u_{h}$ in $x_{j}$ direction with $j=2$ for $i=1$ and $j=1$ for $i=2$. Thus the analysis of Schultz (page 17, [9]) yields

$$
\left\|J_{h i} u_{h}-u_{h}\right\|_{\Omega_{h k}} \leqq C h_{j k}^{2}\left\|\partial^{2} u_{h} / \partial x_{j}^{2}\right\|_{\Omega_{h k}},
$$

from which follows the desired estimation.

The finite element approximation $u_{h} \in S^{h}$ for Eq. (5) is given by 


$$
\left\langle u_{h}, \bar{u}_{h}\right\rangle_{h}=\left(f, \bar{u}_{h}\right)_{\Omega} \quad\left({ }^{\forall} \bar{u}_{h} \in S^{h}\right),
$$

while that for Eq. (6) is by

$$
\left\langle u_{h}, \bar{u}_{h}\right\rangle_{h}=\lambda_{h}\left(u_{h}, \bar{u}_{h}\right)_{\Omega} \quad\left({ }^{\forall} \bar{u}_{h} \in S^{h}\right) .
$$

The following two theorems on the solvability of these approximate equations are easy to prove, and hence presented without proofs.

Theorem 3. The finite element solution $u_{h}$ of Eq. (23) exists uniquely for any $f \in L_{2}(\Omega)$ and satisfies

$$
\left\|u_{h}\right\|_{h} \leqq C\|f\|_{\Omega} .
$$

Theorem 4. The approximate eigenvalue equation (24) has as many eigenvalues and the corresponding eigenfunctions as $L_{h}$, the dimension of $S^{h}$. All the eigenvalues $\left\{\lambda_{h i}\right\}_{i=1}^{L_{h}}$ are positive and they can be arranged as

$$
0<\lambda_{h 1} \leqq \lambda_{h 2} \leqq \cdots<\infty
$$

The eigenfunctions $\left\{u_{h i}\right\}_{i=1}^{L_{n}}$ can be normalized as

$$
\left(u_{h i}, u_{h j}\right)_{\Omega}=\delta_{i j} \quad\left(1 \leqq i, j \leqq L_{h}\right)
$$

\section{Error Estimates for Boundary-value Problem}

This section is to deal with error estimation of the finite element solution for the static problem.

Let us define $u_{h}^{*} \in S^{h}$ for $u \in H_{A}$ by the relation

$$
\left\langle u_{h}^{*}, \bar{u}_{h}\right\rangle_{h}=\left\langle u, \bar{u}_{h}\right\rangle_{h} \quad\left({ }^{\forall} \bar{u}_{h} \in S^{h}\right) .
$$

Such $u_{h}^{*}$ exists uniquely in $S^{h}$ as seen from the discussion of the preceding section. The mapping from $H_{A}$ into $S^{h}$ introduced in this way is denoted by $P_{h}$. Clearly, $\left\|P_{h} u\right\|_{h} \leqq\|u\|$ and

$$
\left\|\bar{u}_{h}-u\right\|_{h}^{2}=\left\|P_{h} u-u\right\|_{h}^{2}+\left\|\bar{u}_{h}-P_{h} u\right\|_{h}^{2}
$$

for any $\bar{u}_{h} \in S^{h}$ and $u \in H_{A}$. Therefore $P_{h} u \in S^{h}$ is the best approximation of $u$ in the following sense: 


$$
\left\|P_{h} u-u\right\|_{h}=\min _{\bar{u}_{h} \in S^{h}}\|\| \bar{u}_{h}-u \|_{h} .
$$

Let us define a bilinear form by

$$
B_{h}\left(u, \bar{u}_{h}\right)=\sum_{i=1}^{2}\left(\partial^{4} u / \partial x_{i}^{4}, \bar{u}_{h}-J_{h i} \bar{u}_{h}\right)_{\Omega}
$$

for $u \in H^{4}(\Omega)$ and $\bar{u}_{h} \in S^{h}$. Then the following holds.

Lemma 5. Let $u$ and $u_{h}$ be the solutions of Eqs. (5) and (23) for the same $f \in L_{2}(\Omega)$, respectively. Then

$$
B_{h}\left(u, \bar{u}_{h}\right)=\left\langle u_{h}-u, \bar{u}_{h}\right\rangle_{h}=\left(f, \bar{u}_{h}\right)_{\Omega}-\left\langle u, \bar{u}_{h}\right\rangle_{h}
$$

for any $\bar{u}_{h} \in S^{h}$.

Proof. Because of the relation $\Delta \Delta u=f$ in $\Omega$ for $u \in H^{4}(\Omega)$ and of the definition of $u_{h}$, it holds that

$$
\begin{aligned}
\left\langle u_{h}, \bar{u}_{h}\right\rangle_{h}=\left(f, \bar{u}_{h}\right)_{\Omega} & \\
=B_{h}\left(u, \bar{u}_{h}\right) & +2\left(\partial^{4} u / \partial x_{1}^{2} \partial x_{2}^{2}, \bar{u}_{h}\right)_{\Omega} \\
& +\sum_{i=1}^{2}\left(\partial^{4} u / \partial x_{i}^{4}, J_{h i} \bar{u}_{h}\right)_{\Omega} .
\end{aligned}
$$

Thanks to sufficient smoothness of $u, \bar{u}_{h}$ and $J_{h i} \bar{u}_{h}$ 's, we can apply the divergence theorem to this equation to show

$$
\left\langle u_{h}, \bar{u}_{h}\right\rangle_{h}=\left\langle u, \bar{u}_{h}\right\rangle_{h}+B_{h}\left(u, \bar{u}_{h}\right)
$$

with the aid of the boundary conditions for $u$ and $\bar{u}_{h}$. This completes the proof.

The following is an extension of a well-known result for non-conforming schemes (see page 174 of Strang and Fix [10]).

Lemma 6. Let $u$ and $u_{h}$ be the exact solutions of Eqs. (5) and (23), respectively. Then

$$
\left\|u_{h}-u\right\|_{h}^{2}=\left\|P_{h} u-u\right\|_{h}^{2}+A_{h}^{2}(u),
$$


where

$$
A_{h}(u)=\sup _{\substack{\bar{u}_{h} \in S^{h} \\ \bar{u}_{h} \neq 0}}\left[\left|B_{h}\left(u, \bar{u}_{h}\right)\right| /\left\|\bar{u}_{h}\right\|_{h}\right]
$$

Proof. Since Eq. (29) holds, we need only to prove $A_{h}(u)=\| u_{h}$ $-P_{h} u \|_{h}$. But this is obvious from the relation $B_{h}\left(u, \bar{u}_{h}\right)=\left\langle u_{h}-P_{h} u\right.$, $\left.\bar{u}_{h}\right\rangle_{h}$ obtained in the preceding lemma.

Now we can easily prove the following two lemmas by the use of the results of the preceding section.

Lemma 7. Let $A_{h}(u)$ be defined by Eq. (34) for $u \in H^{4}(\Omega)$. Then we have from lemma 4 that

$$
A_{h}(u) \leqq C h^{2}|u|_{4, \Omega} .
$$

Lemma 8. Let $u \in H^{4}(\Omega) \cap H_{0}^{1}(\Omega)$. Then we have from lemma 3 and Eq. (30) that

$$
\left\|\left.\left|P_{h} u-u \|_{h} \leqq C h^{2}\right| u\right|_{4, \Omega} .\right.
$$

The following theorem gives error bounds of the finite element solution. It also implies that the Zienkiewicz observation (page 190, [11]) is true.

Theorem 5. Let $u$ and $u_{h} \in S^{h}$ be the solutions of Eqs. (5) and (23), respectively. Then

$$
\begin{gathered}
\left\|e_{h}\right\|_{h} \leqq C h^{2}\|f\|_{\Omega}, \\
\left\|e_{h}\right\|_{1, \Omega} \leqq C h^{2}\|f\|_{\Omega}, \\
\max _{x \in \bar{\Omega}}\left|e_{h}(x)\right| \leqq C h^{2}\|f\|_{\Omega}, \\
\left\|J_{h i} u_{h}-u\right\|_{\Omega} \leqq C h^{2}\|f\|_{\Omega}, \\
\left\|\partial J_{h i} u_{h} / \partial x_{i}-\partial u / \partial x_{i}\right\|_{\Omega} \leqq C h^{2}\|f\|_{\Omega},
\end{gathered}
$$

where $e_{h}=u_{h}-u$ and $i=1,2$. 
Proof. The first estimation follows from the lemmas in this section, while the others may be derived with the aid of lemma 1.

\section{Error Estimates for Eigenvalue Problem}

We will give error estimates to approximation of the first $m$ eigenvalues and the eigenfunctions. Here the positive integer $m$ is of course not greater than $L_{h}$, the dimension of $S^{h}$. Since $S^{h}$ is non-conforming, care should be taken especially in the evaluation of lower bounds of eigenvalues. The explanation of this section is focussed on this subject. In the other aspects, the method of error analysis employed here is essentially the same as done by Strang and Fix (sec. 6.3, [10]), hence the related results will be presented without complete proofs.

We assume that the exact and the approximate eigenfunctions are subjected to Eqs. (10) and (27), and the condition

$$
\left(u_{i}, u_{h i}\right)_{\Omega} \geqq 0 \quad\left(1 \leqq i \leqq L_{h}\right) .
$$

We will use the Rayleigh principle for $\lambda_{i}(1 \leqq i<\infty)$ :

$$
\lambda_{i}=\min _{\substack{u \in E_{i-1} \\ u \neq 0}}\left(\|u\|^{2} /\|u\|_{\Omega}^{2}\right),
$$

and the min-max principle for $\lambda_{h i}\left(1 \leqq i \leqq L_{h}\right)$ :

$$
\lambda_{h i}=\min _{S_{i}^{h} \subset S^{h}} \max _{\substack{u_{h} \in S_{i}^{h} \\ u_{h} \neq 0}}\left(\left\|u_{h}\right\|\left\|_{h}^{2} /\right\| u_{h} \|_{\Omega}^{2}\right) .
$$

In the above, $E_{i}$ is the space spanned by $\left\{u_{j}\right\}_{j=1}^{i}, E_{i}^{\perp}$ its orthogonal complement in $H_{A}$, and $S_{i}^{h}$ an arbitrary $i$-dimensional subspace of $S^{h}$.

Let us define $\tilde{u}_{h i} \in S_{h}\left(1 \leqq i \leqq L_{h}\right)$ by

$$
\left\langle\tilde{u}_{h i}, \bar{u}_{h}\right\rangle_{h}=\lambda_{i}\left(u_{i}, \bar{u}_{h}\right)_{\Omega} \quad\left({ }^{\forall} \bar{u}_{h} \in S^{h}\right),
$$

which is well-defined thanks to Theorem 3. Then the following holds from Theorem 5 since $\left\|u_{i}\right\|_{\Omega}=1$.

Lemma 9. Let $\tilde{u}_{h i}$ be the approximation of $u_{i}$ defined above. Then $\tilde{u}_{h i}-u_{i}$ satisfies the same error estimates as hold for $u_{h}-u$ in 
Theorem 5, if $\|f\|_{\Omega}$ is replaced with $\lambda_{i}$.

As a result, $\left\{\tilde{u}_{h i}\right\}_{i=1}^{m}$ is linearly independent for sufficiently small $h$. The subspace of $S^{h}$ spanned by $\left\{\tilde{u}_{h j}\right\}_{j=1}^{i}$ will be denoted by $E_{i}^{h}$.

Lemma 10. Let $u_{h} \in E_{i}^{h}$ be expressed by $u_{h}=\sum_{j=1}^{i} a_{j} \tilde{u}_{h j}$. Then

$$
\begin{gathered}
\left\|u_{h}\right\|\left\|_{h}^{2}=\right\| u\left\|^{2}+2 B_{h}\left(u, u_{h}\right)-\right\| u_{h}-u \|_{h}^{2}, \\
\left\|u_{h}\right\|_{\Omega}^{2}=\|u\|_{\Omega}^{2}+2\left\langle u^{\dagger}-u_{h}^{\dagger}, u_{h}-u\right\rangle_{h}+2 B_{h}\left(u^{\dagger}, u_{h}\right) \\
+2 B_{h}\left(u, u_{h}^{\dagger}\right)_{\Omega}+\left\|u_{h}-u\right\|_{\Omega}^{2}, \\
u=\sum_{j=1}^{i} a_{j} u_{j} \in E_{i}, \quad u^{\dagger}=\sum_{j=1}^{i} \frac{a_{j}}{\lambda_{j}} u_{j} \in E_{i} \\
u_{h}^{\dagger}=\sum_{j=1}^{i} \frac{a_{j}}{\lambda_{j}} \tilde{u}_{h j} \in E_{i}^{h} .
\end{gathered}
$$

where

and

Proof. The above follows from Eqs. (6), (32) and (41). For example, Eq. (42) may be derived by substituting the relation $\left\langle u_{h}-u, u_{h}\right\rangle_{h}$ $=B_{h}\left(u, u_{h}\right)$ into the identity

$$
\|u\|^{2}=\left.\left\|u_{h}\right\|\right|_{h} ^{2}-2\left\langle u_{h}-u, u_{h}\right\rangle_{h}+\left\|u_{h}-u\right\|_{h}^{2} .
$$

(cf. Lemmas 6.1 and 6.2 of Strang and Fix [10].)

Lemma 11. Let $u_{h}$ be an arbitrary non-zero element of $S_{i}^{h}(1$ $\left.\leqq i \leqq L_{h}\right)$ such that

$$
\left(u_{h}, u_{j}\right)_{\Omega}=0 \quad(1 \leqq j \leqq i-1)
$$

Such $u_{h}$ exists since $S_{i}^{h}$ is i-dimensional and the number of constraints in Eq. (44) is $i-1$. When $i=1$, no constraint is imposed on $u_{h}$. Define $u \in H_{A}$ by

$$
\langle u, \bar{u}\rangle=\left(u_{h}, \bar{u}\right)_{\Omega} \quad\left({ }^{\forall} \bar{u} \in H_{A}\right) .
$$

Then $u \in E_{i-1}^{\perp}$, and

$$
\|u\|\|\leqq\| u_{h}\left\|_{\Omega} / \sqrt{\lambda_{i}}, \quad A_{h}(u) \leqq C h^{2}\right\| u_{h} \|_{\Omega} .
$$


Proof. $u \in E_{i-1}^{\perp}$ since $\left\langle u, u_{j}\right\rangle=\left(u_{h}, u_{j}\right)_{\Omega}=0$ for $1 \leqq j \leqq i-1$. Equating $\bar{u}$ to $u$ in Eq. (45) yields

$$
\|u\|^{2} \leqq\left\|u_{h}\right\|_{\Omega}\|u\|_{\Omega} \leqq\left\|u_{h}\right\|_{\Omega} \frac{1}{\sqrt{\lambda_{i}}}\|u\|
$$

with the aid of the Schwarz inequality and Eq. (39). Thus the former of Ineq. (46) is established, while the latter is obvious from Lemma 7.

Now we can give error estimation to the eigenvalues.

Theorem 6. Let $\lambda_{h i}$ be the approximate eigenvalue of $\lambda_{i}(1 \leqq i \leqq m)$. Then, for sufficiently small $h$, it holds that

$$
\left|\lambda_{h i}-\lambda_{i}\right| \leqq C h^{2}
$$

where $C$ can be taken to be dependent only on $m$.

Proof. To obtain an upper bound of $\lambda_{h i}$, we employ the notations and the results of Lemma 10 , to which we add a condition $\|u\|_{\Omega}$ $=1$. Then, we can easily see that $\|u\|^{2} \leqq \lambda_{i},\|\Delta \Delta u\|_{\Omega} \leqq \lambda_{i},\left\|u^{\dagger}\right\| \| 1$, $\left\|\Delta \Delta u^{\dagger}\right\|_{\Omega} \leqq 1$, and

$$
\left\langle u_{h}, \bar{u}_{h}\right\rangle_{h}=\left(\Delta \Delta u, \bar{u}_{h}\right)_{\Omega}, \quad\left\langle u h_{h}^{\dagger}, \bar{u}_{h}\right\rangle_{h}=\left(\Delta \Delta u^{\dagger}, \bar{u}_{h}\right)_{\Omega}
$$

for any $\bar{u}_{h} \in S^{h}$. Therefore, we have, from the discussion of the preceding section, that

$$
\begin{gathered}
\left\|u_{h}-u\right\|_{h} \leqq C h^{2} \lambda_{i}, \quad\left\|u_{h}^{\dagger}-u^{\dagger}\right\|_{h} \leqq C h^{2} \\
\left\|u_{h}-u\right\|_{\Omega} \leqq C h^{2} \lambda_{i}, \quad A_{h}(u) \leqq C h^{2} \lambda_{l}, \\
A_{h}\left(u^{\dagger}\right) \leqq C h^{2} .
\end{gathered}
$$

Applying these estimates to $\left\|u_{h}\right\|\left\|_{h}^{2} /\right\| u_{h} \|_{\Omega}^{2}$ with the aid of Eqs. (42) and (43), we find

$$
\max _{\substack{u_{h} \in E_{i}^{h} \\ u_{h} \neq 0}}\left(\left\|u_{h}\right\|\left\|_{h}^{2} /\right\| u_{h} \|_{\Omega}^{2}\right) \leqq \lambda_{i}+C^{*} h^{2}\left(\lambda_{i}^{\frac{1}{2}}+\lambda_{i}+\lambda_{i}^{\frac{3}{2}}\right)
$$


for sufficiently small $h$. This is an upper bound of $\lambda_{h i}$ because of the min-max principle.

To obtain a lower bound, we use the same $u_{h}$ and $u$ as in Lemma 11. From Lemma 5, we can show that they satisfy the relation

$$
\left\|u_{h}\right\|_{\Omega}^{2}=\left\langle u, u_{h}\right\rangle_{h}+B_{h}\left(u, u_{h}\right) \leqq\left(\|u\|+A_{h}(u)\right)\left\|u_{h}\right\|_{h},
$$

from which follows

$$
\left\|u_{h}\right\|_{\Omega}^{2} \leqq\left(\lambda_{i}^{-\frac{1}{2}}+C h^{2}\right)\left\|u_{h}\right\|_{\Omega}\left\|u_{h}\right\|_{h}
$$

with the aid of the estimates (46). Thus, we have, for sufficiently small $h$,

$$
\lambda_{h i} \geqq \lambda_{i}\left(1-C^{* *} h^{2} \sqrt{\lambda_{i}}\right)
$$

from the min-max principle. This completes the proof.

Next, we should give error estimates to eigenfunctions.

Lemma 12. Let $\left\{\tilde{u}_{h i}\right\}_{i=1}^{L_{h}}$ be defined by Eq. (41). Then

$$
\begin{gathered}
\left\|u_{h i}-\tilde{u}_{h i}\right\|_{h}^{2}=\left(\lambda_{h i}-\lambda_{i}\right)\left(u_{h i}, u_{h i}-\tilde{u}_{h i}\right)_{\Omega} \\
+\lambda_{i}\left(u_{h i}-u_{l}, u_{h i}-\tilde{u}_{h l}\right)_{\Omega}, \\
\lambda_{i}\left(u_{i}, u_{h J}\right)_{\Omega}=\lambda_{h j}\left(u_{h j}, \tilde{u}_{h i}\right)_{\Omega}=\left\langle\tilde{u}_{h i}, u_{h j}\right\rangle_{h}
\end{gathered}
$$

for $1 \leqq i, j \leqq L_{l l}$.

Proof. All of these follows from the definitions of $u_{h i}$ and $\tilde{u}_{h i}$. (cf. Lemmas 6.3 and 6.4 of Strang and Fix [10]).

Theorem 7. We assume that $h$ is small enough and $1 \leqq i \leqq m$. When there is no repeated eigenvalue in $\lambda_{i}$ 's, $u_{h i}-u_{i}$ satisfies the same error estimates as $u_{h}-u$ in Theorem 5, if $\|f\|_{\Omega}$ is replaced with a suitable constant dependent only on $m$. When there is a repeated eigenvalue, we can choose $\left\{u_{i}\right\}_{i=1}^{L_{h}}$ so that $u_{h i}-u_{i}$ satisfies the same error estimates.

Proof. As seen from Eq. (48), the cssence of the proof lies in 
the estimation of $\left\|u_{h i}-\tilde{u}_{h i}\right\|_{\Omega}$ or $\left\|u_{h i}-u_{i}\right\|_{\Omega}$, for which Eq. (49) may be effectively employed. The details of the proof are almost the same as used by Strang and Fix (sec. 6.3 [10]).

\section{Numerical Experiments}

Some numerical experiments are conducted to show the validity of the error analysis given in the preceding sections. In the sequel, $\Omega$ is chosen a square defined by $\left|x_{i}\right|<1 / 2(i=1,2)$. All the computations are performed by the double precision arithmetic on HITAC $5020 \mathrm{~F}$ computer, and the $5 \times 5$ product Gauss quadrature formula is employed for the integrations in each finite element.

\subsection{Boundary-value Problem.}

We first analyze a simply supported square plate under lateral loading $f(x)=4 \pi^{4} \cos \left(\pi x_{1}\right) \cos \left(\pi x_{2}\right)$, for which the exact solution is

$$
u(x)=\cos \left(\pi x_{1}\right) \cos \left(\pi x_{2}\right) .
$$

The square is divided into $n \times n$ uniform mesh, the results being obtained for several values of $n$. The Gauss elimination method is employed to solve the linear simultaneous equations.

Table 1 shows the convergence character of $e_{h}=u_{h}-u$ against $h=1 / n$ measured by the maximum norm, $L_{2}$-norm and \|\|$\|_{h}$. Clearly, all of these are asymptotically proportional to $h^{2}$, as predicted by the

Table. 1. Convergence of $e_{h}=u_{h}-u$ for the boundary-value problem.

\begin{tabular}{|l|c|c|c|}
\hline \multicolumn{1}{|c|}{$h$} & $\max _{x \in \bar{\Omega}}\left|e_{h}(x)\right| / h^{2}$ & $\left\|e_{h}\right\|_{\Omega} / h^{2}$ & $\left\|e_{h}\right\|_{h} / h^{2}$ \\
\hline $1 / 2$ & 0.726 & 0.274 & 8.44 \\
$1 / 4$ & 0.804 & 0.374 & 8.78 \\
$1 / 6$ & 0.815 & 0.394 & 8.84 \\
$1 / 8$ & 0.818 & 0.402 & 8.86 \\
$1 / 10$ & 0.820 & 0.405 & 8.87 \\
$1 / 20$ & 0.822 & 0.410 & 8.89 \\
\hline
\end{tabular}


theory. It is to be noted that the convergence rate of the deflection is not better than that of moments unlike in the compatible models. In other words, Nitsche's trick does not work in this problem.

\subsection{Eigenvalue Problem.}

As a second example, we treat the first approximate eigenvalue $\lambda_{h 1}$ and the corresponding eigenfunction $u_{h 1}$. The exact ones are, as well known,

$$
\lambda_{1}=4 \pi^{4} \quad \text { and } \quad u(x)=\cos \left(\pi x_{1}\right) \cos \left(\pi x_{2}\right)
$$

In the calculation, the square is again decomposed into $n \times n$ mesh, and $u_{h 1}$ is obtained under the normalizing conditions

$$
\left\|u_{h 1}\right\|_{\Omega}=\left\|u_{1}\right\|_{\Omega}=1 / 2 \quad \text { and } \quad\left(u_{h 1}, u_{1}\right)_{\Omega} \geqq 0 \text {. }
$$

The approximate characteristic equations are solved by the subspace iteration method with two trial vectors.

Tables 2 and 3 are on the convergence characters of $\lambda_{h 1}$ and $e_{h 1}$ $=u_{h 1}-u_{1}$, respectively. Clearly, $\left|\lambda_{h 1}-\lambda_{1}\right|=O\left(h^{2}\right)$, as predicted from the theory, and $\lambda_{h 1}$ approaches $\lambda_{1}$ from below as $h$ tends to 0 unlike in the case of compatible models. It is here to be noticed that the error of the approximate eigenvalue is usually $O\left(h^{4}\right)$ in compatible models when the error of the moments is $O\left(h^{2}\right)$ (see sec. 6.3 of Strang and Fix [10]). It is quite interesting that the order of the deflection error

Table 2. Convergence of the first eigenvalue.

\begin{tabular}{|c|c|c|}
\hline \multicolumn{1}{|c|}{$h$} & $\lambda_{h 1}$ & $\left(\lambda_{1}-\lambda_{h 1}\right) / h^{2}$ \\
\hline $1 / 2$ & 343.766 & 183.5 \\
$1 / 4$ & 372.252 & 278.2 \\
$1 / 6$ & 381.282 & 300.8 \\
$1 / 8$ & 384.805 & 309.2 \\
$1 / 10$ & 386.504 & 313.2 \\
$1 / 20$ & 388.840 & 318.6 \\
\hline Exact & 389.636 & $\ldots \ldots$. \\
\hline
\end{tabular}

Table 3. Convergence of $\mathrm{e}_{h_{1}}=\mathrm{u}_{h_{1}}-\mathrm{u}_{1}$ for the eigenvalue problem.

\begin{tabular}{|c|c|c|c|}
\hline$h$ & $\max _{x \in \bar{\Omega}}\left|e_{h 1}(x)\right| / h^{4}$ & $\left\|e_{h 1}\right\|_{\Omega} / h^{4}$ & $\left\|e_{h 1}\right\|_{h} / h^{2}$ \\
\hline $1 / 2$ & 0.70 & 0.245 & 7.91 \\
$1 / 4$ & 0.87 & 0.268 & 8.58 \\
$1 / 6$ & 0.98 & 0.276 & 8.75 \\
$1 / 8$ & 1.02 & 0.279 & 8.81 \\
$1 / 10$ & 1.04 & 0.281 & 8.84 \\
$1 / 20$ & 1.07 & 0.283 & 8.88 \\
\hline
\end{tabular}


is $O\left(h^{4}\right)$ unlike in the static problem. This is because $u_{h 1}$ coincides with $\hat{u}_{h 1}$, the interpolate of $u_{1}$, in shape (but not in value), whose maximum and $L_{2}$-errors are both $O\left(h^{4}\right)$. (Notice that $u_{h 1}=\frac{1}{2} \hat{u}_{h 1} /$ $\left\|\hat{u}_{h 1}\right\|_{\Omega}$ from this fact and the normalizing conditions. Then the observed orders may be easy to check.)

\section{Concluding Remarks}

The convergence of the ACM non-conforming scheme has been discussed. Almost all the results in this paper hold for plates with clamped edge conditions, so long as the exact solution is sufficiently smooth and the shape of domain is exactly represented by rectangular elements.

As well known, non-conforming method may or may not converge, and the penalty method proposed by Babuska and Zlamal [1] is an effective technique to assure the convergence. However, its use appears to be less necessary in the present finite element model. The difficulty of the convergence proof lies essentially in the evaluation of the second term in Eq. (33). Although the patch test may be conveniently used for this purpose in special type of finite elements (Strang and Fix [10]), no general theory appears to be available at present. The techniques developed in this paper are not general enough, but may offer an effective tool to certain type of finite elements.

The author would like to express his deepest appreciation to Prof. T. Ushijima, University of Electro-Communications, for his valuable advices. This work was supported by the Grant-in-Aid for Scientific Research from Ministry of Education and the Sakkokai Foundation.

\section{References}

[1] Babuska, I. and Zlamal, M., Nonconforming elements in the finite element method with penalty, SIAM J. Numer. Anal., 10 (1973) 863-875.

[2] Ciarlet, P. G., Conforming and nonconforming finite element methods for solving the plate problem, Conference on the Numerical Solution of Differential Equations, pp. 21-31, Springer, Berlin, 1974.

[3] Hall, C. A. and Kennedy, J. W., Convergence of a Ritz approximation for the steady state heat flow problem, J. Inst. Math. Appl. 12 (1973) 187-196. 
[4] Melosh, R. J., A stiffness matrix for the analysis of thin plates in bcnding, J. Aero-Space Sci., 28 (1961) 34-42.

[5] Melosh, R.J., Basis of derivation of matrices for the direct stiffness method, AIAA J., 1 (1963) 1631-1637.

[6] Mikhlin, S. G., Variational methods in mathematical physics (in Russian), Nauka, Moskow, 1965.

[7] Miyoshi, T., Convergence of finitc element solutions represented by a nonconforming basis, Kumamoto J. Sci. (Nath.), 9 (1972) 11-20.

[8] Mizohata, S., Theory of partial differential equations, Cambridge University Press, London, 1973.

[9] Schults, M. H., Spline Analysis, Prentice-Hall, New York, 1973.

[10] Strang, G. and Fix, G., An analysis of the finite element method, Prentice-Hall, New York, 1973.

[11] Zienkiewicz, O.C., The finite element method in enginecring science, McGraw-Hill, London, 1971. 
